\title{
Variation in ITGB3 is associated with whole-blood serotonin level and autism susceptibility
}

\author{
Lauren A Weiss ${ }^{1,6}$, Gülüm Kosova ${ }^{2}$, Ryan J Delahanty ${ }^{3}$, Lan Jiang ${ }^{3}$, Edwin H Cook Jr ${ }^{4}$, \\ Carole Ober ${ }^{1}$ and James S Sutcliffe ${ }^{*, 3,5}$
}

${ }^{1}$ Department of Human Genetics, The University of Chicago, Chicago, IL, USA; ${ }^{2}$ Committee on Genetics, The
University of Chicago, Chicago, IL, USA; ${ }^{3}$ Department of Molecular Physiology and Biophysics, Vanderbilt University,
Nashville, TN, USA; ${ }^{4}$ Department of Psychiatry, University of Illinois at Chicago, Chicago, IL, USA; ${ }^{5}$ Center for
Molecular Neuroscience and Vanderbilt Kennedy Center, Vanderbilt University, Nashville, TN, USA

Autism is a pervasive developmental disorder affecting more males than females. Heritability estimates for autism can rise above $\mathbf{9 0 \%}$, and genes influencing the serotonin system are strong candidates for autism susceptibility genes, as drugs selectively acting on the serotonin system are some of the most effective treatments for maladaptive behaviors seen in autism. ITGB3 was recently identified as a male quantitative trait locus (QTL) for whole-blood serotonin levels in the Hutterites $(P=0.0003)$. Here, we demonstrate associations between variation in ITGB3 and serotonin levels in two outbred samples $(P=0.010$ and 0.015$)$. Lastly, we show that a coding variant of ITGB3 is associated with autism susceptibility in a large multiplex sample $(P=\mathbf{0 . 0 0 0 8 2})$, and that this variation has different effects in males and females $(P=\mathbf{0 . 0 0 1 8})$. European Journal of Human Genetics (2006) 14, 923-931. doi:10.1038/sj.ejhg.5201644; published online 17 May 2006

Keywords: autism; integrin beta3; serotonin; 5-HT; quantitative trait locus; platelet

\section{Introduction}

Autism is a pervasive developmental disorder that impairs reciprocal social interaction and communication, and is associated with restricted and repetitive behaviors. ${ }^{1}$ Narrowly defined 'autistic disorder' has an estimated prevalence of approximately one in 500 children, although as many as one in 166 children may present on the broader autism spectrum. ${ }^{2,3}$ Approximately $80 \%$ of individuals with autism are male. Although idiopathic autism is strongly genetic, no common susceptibility alleles have been conclusively implicated, and the underlying biology is poorly understood. ${ }^{4}$ One of the oldest and most

*Correspondence: Dr JS Sutcliffe, Department of Molecular Physiology and Biophysics, Center for Molecular Neuroscience, 702 Light Hall, Vanderbilt University, Nashville, TN 37232-0615, USA.

Tel/Fax: + 1615936 3626; E-mail: james.s.sutcliffe@vanderbilt.edu

${ }^{6}$ Current address: Psychiatric and Neurodevelopmental Genetics Unit, Center for Human Genetic Research, Massachusetts General Hospital, Harvard Medical School, Boston, MA, USA

Received 20 January 2006; revised 28 March 2006; accepted 4 April 2006; published online 17 May 2006 replicated findings is hyperserotonemia, or elevated platelet serotonin levels, in about $30 \%$ of individuals with autism. 5,6 Moreover, subjects with autism who have an affected sibling show higher levels of platelet serotonin compared with subjects without an affected sibling. ${ }^{7}$ Several studies have revealed positive correlation of serotonin levels between affected individuals and their first-degree (unaffected) relatives $(0.50<r<0.71)$, further suggesting the heritability of this trait. ${ }^{8,9}$ In addition, selective serotonin reuptake inhibitors (SSRIs) and atypical antipsychotic medications, which are often effective in autism to treat disruptive anxiety-related behaviors and aggression, respectively, act on the serotonin system. ${ }^{10,11}$ Data thus suggest that at least some of the serotonin dysregulation in autism families is not secondary to autism, but rather may inform us about a heritable predisposing factor.

Functional variants at the serotonin transporter locus (SLC6A4) have been associated with autism in some studies, ${ }^{12,13}$ but these polymorphisms do not account for 
all of the variation in serotonin levels among the autism population. ${ }^{14-17}$ Recently, the $\beta 3$-integrin gene (ITGB3) was identified as a quantitative trait locus (QTL) for wholeblood serotonin levels, primarily in male subjects as compared with female subjects. ${ }^{18,19}$ This finding implicates a locus with no previously recognized role in serotonin regulation. We hypothesized that more detailed analysis of ITGB3 might reveal functional variation, clarify the mechanism underlying its association with serotonin levels, and explain the sex-specific effects in association. We previously showed that variation in ITGB3 is associated with endophenotypes for common diseases, such as cardiovascular disease $^{20}$ and asthma, ${ }^{21}$ whose etiologies may be influenced by serotonin physiology. However, serotonin is best known for its role as a neurotransmitter important in central nervous system function and psychiatric disease. Thus, an important aim of our study was to test for association between ITGB3 and autism.

The ITGB3 gene has 15 exons and spans $60 \mathrm{~kb}$ on chromosome $17 \mathrm{q} 21.3$, about $20 \mathrm{cM}$ distal to SLC6A4. The encoded $\beta 3$ integrin is a subunit of the platelet- and megakaryocyte-specific heterodimeric fibrinogen receptor and the widely expressed heterodimeric vitronectin receptor. In addition to roles in platelet activation and adhesiondependent tissue modeling, integrin receptors have been shown to have signaling roles that can influence transcription and translation. ${ }^{22-24}$ The ITGB3 Leu33Pro singlenucleotide polymorphism (SNP), encoding the platelet antigen $\mathrm{Pl}^{\mathrm{A} 1 / \mathrm{A} 2}$ (or HPA-1a/1b), is associated with functional properties of fibrinogen receptors, such as differential platelet adhesion and aggregation. ${ }^{25-27}$ This SNP was identified as a QTL for whole-blood serotonin level in a genome-wide association study in the Hutterites, a young founder population of European descent. ${ }^{19}$ However, variation in the gene other than Leu33Pro had not been comprehensively evaluated in this or other populations. Thus, the aims of this study were to (1) fine map the association between serotonin level and ITGB3 in the Hutterites, (2) evaluate associations between ITGB3 alleles and serotonin levels in outbred populations, and (3) test for association between variation in ITGB3 and autism.

\section{Materials and methods Hutterites}

Details of the Hutterite founder population, sampling strategy, and the utility of this population for mapping complex traits have been described previously. ${ }^{19,28,29}$ Briefly, whole-blood serotonin was measured in 567 Hutterites (300 female and 267 male subjects), who are related in a 1623-person pedigree, ${ }^{28}$ after obtaining informed consent. ${ }^{19,29}$ The distribution of the serotonin levels $(191 \pm 79 \mathrm{ng} / \mathrm{ml}$; male subjects: $174 \pm 76 \mathrm{ng} / \mathrm{ml}$; female subjects: $206 \pm 79 \mathrm{ng} / \mathrm{ml}$ ) was normalized by $\ln$ transformation in order to use statistical tools relying on a normal distribution. These studies were approved by The University of Chicago and University of South Dakota Institutional Review Boards.

\section{Chicago controls}

We measured whole-blood serotonin level in healthy outbred adults ( $>18$ years) who were ascertained as controls for genetic studies of asthma. The control subjects reported either three or four grandparents of European ancestry $(N=206)$ or of African or African-American ancestry $(N=186)$. Serotonin levels were measured, using methods described for the Hutterite samples, ${ }^{19}$ in a subset of the controls corresponding to 56 European Americans (14 male subjects: $179 \pm 67 \mathrm{ng} / \mathrm{ml} ; 42$ female subjects: $172 \pm 74 \mathrm{ng} / \mathrm{ml}$ ) and 36 African Americans (11 male subjects: $220 \pm 93 \mathrm{ng} / \mathrm{ml} ; 25$ female subjects: $200 \pm 87 \mathrm{ng} / \mathrm{ml})$.

\section{Chicago autism sample}

The Chicago autism sample consisted of 87 outbred Caucasian trios (affected child and parents). Recruitment, assessment, and inclusion criteria were the same as those outlined by Kim et al, ${ }^{30}$ but only one sibling was randomly selected from each affected sibling pair, and subjects (1) had sufficient blood or DNA available for fine mapping studies and (2) met Autism Diagnostic Interview-Revised $\left(A D I-R^{31}\right)$ and Autism Diagnostic Observation Schedule (ADOS- $\mathrm{G}^{32}$ ) criteria for classification of autistic disorder. The samples from which serotonin was measured (and not reported previously) corresponded to 50 Caucasian autism probands (age $=5.9 \pm 3.3$ years) from The University of Chicago who were not taking psychotropic medications (39 male and 11 female subjects). Whole-blood serotonin was measured by high-performance liquid chromatography (using methods described for the Hutterites ${ }^{19}$ ), and the distribution of levels was $244 \pm 81 \mathrm{ng} / \mathrm{ml}$ for male subjects, $222 \pm 64 \mathrm{ng} / \mathrm{ml}$ for female subjects, and $239 \pm 77 \mathrm{ng} / \mathrm{ml}$ overall.

\section{Vanderbilt autism sample}

The Vanderbilt autism sample consisted of 564 multiplex families and 79 trios collected from different sites. Of this sample, 327 families were from AGRE, 101 were from the Tufts-Vanderbilt University consortium, 85 were from Iowa, and 130 were from Stanford. The AGRE, Iowa, and Stanford samples were obtained from the NIMH Center for Collaborative Genetic Studies on Mental Disorders. Multiplex families were selected by presence of a single proband who met full Autism Diagnostic Interview-Revised (ADI-R) criteria for autism and a second sibling with either autism or on the broader spectrum. Analysis included 79 trios in addition to the multiplex families. Of the 1255 affected individuals, 987 (79\%) were male and 268 (21\%) were female. Caucasian individuals made up $80 \%$ of the sample, 9\% were of unknown ethnicity, and 11\% were 
Hispanic-Latino, African American, Asian, Native Hawaiian or Pacific Islander, multi-ethnic, or 'other'. Serotonin levels were not obtained for these families, as they were ascertained strictly for genetic studies.

\section{Sequencing}

In order to identify common variation in the exons and splice junctions of ITGB3, we selected 12 Hutterite subjects representing all ITGB3 haplotypes $>1 \%$, defined by the 15 SNPs previously genotyped in this population. ${ }^{20}$ Primers were designed based on the reference sequence (hg16) to amplify all exons and $>45 \mathrm{bp}$ of flanking sequence, $1400 \mathrm{bp}$ of the region $5^{\prime}$ to exon 1 , and one region each in introns 2 and 13 identified as conserved noncoding sequences by the Comparative Genomic Resources for Cardiovascular Research using VISTA. Primer sequences and sequencing protocols are available upon request. Sequencing reactions were performed with BigDye v.3.1 (Applied Biosystems, Foster City, CA, USA) and resolved on an ABI 3100. Denaturing high-performance liquid chromatography was used to rule out sequence variation in eight instances $(<3 \%$ amplicons) when sequencing reactions repeatedly failed. DNA sequence was assembled and analyzed using PolyPhred. ${ }^{33}$

\section{Genotyping}

Markers previously genotyped in the Hutterite population include (1) the Leu33Pro variant from the genome screen, ${ }^{19}$ (2) an additional 14 SNPs spanning ITGB3 and $10 \mathrm{~kb}$ of flanking sequence, and (3) markers in flanking genes to the $5^{\prime}$ (MYL4, two SNPs) and 3' (FLJ40342, seven SNPs) of ITGB3. ${ }^{20}$ Four additional SNPs identified from sequencing studies were genotyped in the Hutterite samples (see Results). All 19 ITGB3 SNPs that were genotyped in the Hutterites were genotyped in the Chicago controls (Supplementary Table 2). Intermarker linkage disequilibrium (LD) was measured using the $r^{2}$ statistic, as implemented on the Innate Immunity Programs for Genomics Applications website. SNPs were chosen and genotyped in the autism family samples based in part on results from Hutterite and outbred control studies (SNPs 1, 4,8 , and 11), and in part based on Caucasian HapMap data as tags for common $(\geqslant 5 \%)$ haplotypes across the transcriptional unit (SNPs 1, 16, and 20-23). All genotyping was performed blinded to sample identity and affection status. Genotyping methods for each SNP are shown in Supplementary Table 3.

\section{Statistical analysis}

All genotype data were assessed for Hardy-Weinberg equilibrium. An association test for quantitative phenotypes that takes into account relationships among individuals was used to analyze Hutterite data. In this test, the effect of an allele was represented as a main effect in a linear model, as described previously. ${ }^{18,34}$ The multipoint allele-specific homozygosity by descent test relies on the existence of regions that are homozygous by descent in inbred individuals to detect QTLs that act in a recessive manner. Age and sex were included as covariates in these analyses (see Ober et $a^{29}$ for details) and significance was assessed using a permutation test. ${ }^{34}$

To estimate effect size of associated alleles in the Hutterites, we performed a generalized linear regression of the transformed phenotype values on the covariates, using the estimated covariance matrix (obtained as described by Abney et $a l^{34}$ ) as weights in the Hutterite analysis. We performed this twice for each allele we tested, once under the null hypothesis, with only age and sex as covariates, and once under the alternative hypothesis, with genotype data included as an additional covariate. To estimate the percent variance explained by an allele, we calculated the residual sum of squares (RSS) for each regression and used the equation $\left(\mathrm{RSS}_{\text {null }}-\mathrm{RSS}_{\mathrm{alt}}\right) / \mathrm{RSS}_{\text {null }}$.

Association tests of serotonin levels in the control and autism samples were performed by linear regression using an additive model at each SNP. Age and sex were used as covariates in the analysis of autism subjects. Age was used as a covariate in the outbred controls, but sex and race were not significant covariates in this sample $(P>0.1)$. In addition, by looking at the distribution of serotonin level by genotype in each group, we determined that allelic relationships with serotonin did not differ between Caucasians and African Americans at each SNP; we therefore combined the overall sample for analysis. The transmission disequilibrium test (TDT), as implemented by Genehunter, ${ }^{35}$ was performed to test for joint linkage and association in the Chicago autism trios. A family-based association test, as implemented in $\mathrm{FBAT}^{36}{ }^{36}$ was performed to test for association in the Vanderbilt autism sample, initially under an additive model and subsequently under a dominant model. Because part of this sample overlapped with samples showing linkage in the region, we also reanalyzed association using FBAT taking linkage into account. ${ }^{37}$ Sex differences were assessed using the program MODEL as implemented in an analysis module of the Genetic Power Calculator. It uses a log-linear approach to fit genetic models to multiple TDT samples and test for equal effects across samples (female affected $v s$ male affected, in this case). ${ }^{38}$

\section{Results \\ Resequencing ITGB3 reveals no additional coding variation}

By sequencing individuals representing all common haplotypes in the Hutterites (frequency >1\%) for ITGB3 exons, flanking sequence, and conserved regions, we identified 17 SNPs, including two that were previously undocumented (rs17225109 and rs17218711). Four of the 17 SNPs were not among those we had previously 


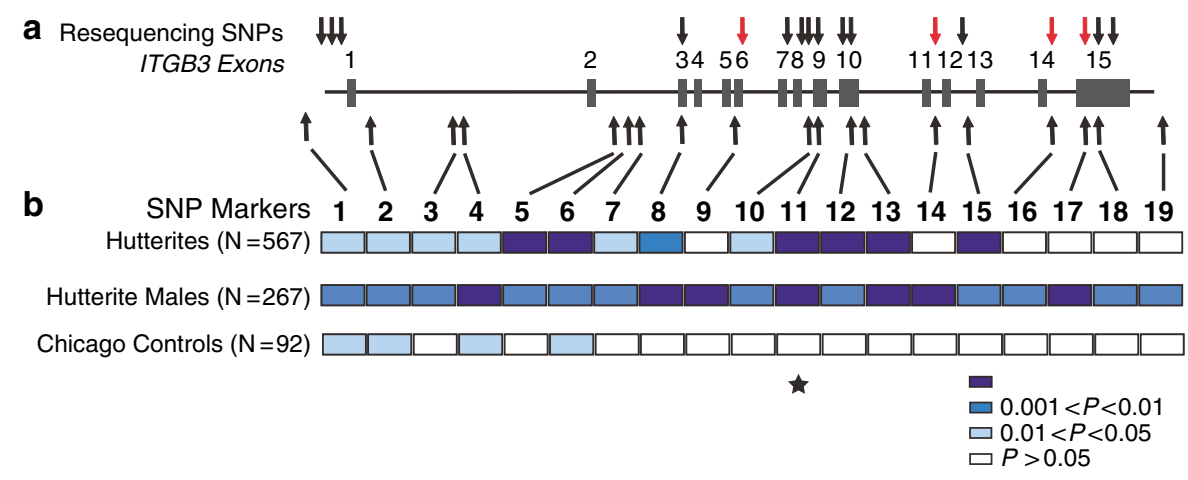

Figure 1 (a) ITGB3 sequencing results. Arrows above the ITGB3 gene structure schematic indicate positions of polymorphisms identified by sequencing of common haplotypes in the Hutterites. Red arrows indicate the subset of those variants subsequently genotyped in the complete sample. (b) Association of ITGB3 alleles with serotonin levels. Arrows below the gene structure schematic indicate SNPs that were genotyped in the complete Hutterite and Chicago control samples, and the shading of boxes below these arrows reflects $P$-values corresponding to association at each SNP. The outbred control sample was predominantly female (67 of 92), thus power-limited sex-specific analyses are not shown. Based on these results, four SNPs $(1,4,8$, and 11$)$ were genotyped in the predominantly male (39 of 50$)$ Chicago autism sample. The SNP associated in this sample $(P<0.05)$ is designated with a star below the $P$-value schematic. Linear regression using an additive model was used in both outbred samples.

genotyped in the Hutterites ${ }^{20}$ and identified new haplotypes; these were genotyped in the Hutterite sample (SNPs 9, 14, 16, and 17; Figure 1a). None of the variation we observed, except the previously genotyped Leu33Pro, ${ }^{19}$ changed the coding sequence or splice donor/acceptor sites in ITGB3. Of the remaining 16 SNPs, four were silent SNPs in exons, three were in the $5^{\prime}$ region, six were intronic, and three were in the $3^{\prime}$-untranslated region (Figure 1a).

\section{Noncoding SNPs in ITGB3 are associated with serotonin level in the Hutterites, controls, and autism patients}

Although many of the ITGB3 SNPs show strong associations to serotonin level in the Hutterites (Figure 1b), we previously reported that none of the SNPs that were genotyped in the flanking genes, MYL4 or FLJ40342, are associated. $^{20}$ This is consistent with strong LD between a number of SNPs across ITGB3 in the Hutterites (eg SNPs 5 and 13), but negligible LD between genes (Figure 2). Because the linkage and association signals at ITGB3 Leu33Pro that were detected in our genome scan were present predominantly in male subjects, ${ }^{18}$ we next performed these analyses in the male Hutterites only (Figure 1b). Every ITGB3 SNP shows strong association $\left(P_{2 \text {-tail }}<0.01\right)$ with serotonin levels in this multipoint test, and for each SNP in ITGB3 except one (SNP 19), the common allele was associated with decreased levels. Because many SNPs within ITGB3, but none in the flanking genes, were associated with serotonin, the region of association was narrowed to ITGB3. However, we could not refine our association signal to a smaller subset of SNPs in the Hutterites.

We next examined whole-blood serotonin levels in an outbred sample of healthy Chicago Caucasian and African-
American controls who were previously genotyped for SNPs in ITGB3. ${ }^{21}$ The Chicago control sample was, by chance, largely female (67 of 92 individuals), although the ITGB3 association in the Hutterites was more significant in male subjects. In the Chicago control sample, four SNPs near the $5^{\prime}$ end of ITGB3 were associated with serotonin levels with $P_{1 \text {-tail }}<0.05$, and the strongest associations were detected with SNPs 1 and $4\left(P_{1-\text { tail }}=0.010\right.$ and 0.011 , respectively; Figure 1b). Nonetheless, common alleles of ITGB3 were associated with relatively lower whole-blood serotonin levels, as in the Hutterites. We verified that the female subjects contributed to this signal, as removing them left no significant association (data not shown). These $P$-values are only nominally significant, considering that we tested 19 SNPs in ITGB3, some of which were in strong LD (making Bonferroni correction conservative) (Figure 2).

An autism sample served as the second replication sample for our studies of ITGB3 and whole-blood serotonin levels. This sample reflects the large male bias in autism (39 of 50 subjects were male). Five SNPs were selected for genotyping in this sample based on results in the Hutterites and Chicago controls: SNPs 1, 8, 11, 14, and 18. In this sample, the common allele at the silent SNP in exon 9 (SNP 11) was associated with lower serotonin levels $\left(P_{1-\text { tail }}=0.015\right.$; Figure $\left.1 \mathrm{~b}\right)$. Interestingly, this association in a largely male sample of individuals with autism was not driven by the hyperserotonemic individuals in the sample (data not shown), rather it was consistent with the multipoint results in male Hutterites (Figure 1), where the common allele at SNP 11 showed the most significant association with lower whole-blood serotonin levels, and is the third independent sample in which noncoding variation in ITGB3 is associated with serotonin. 


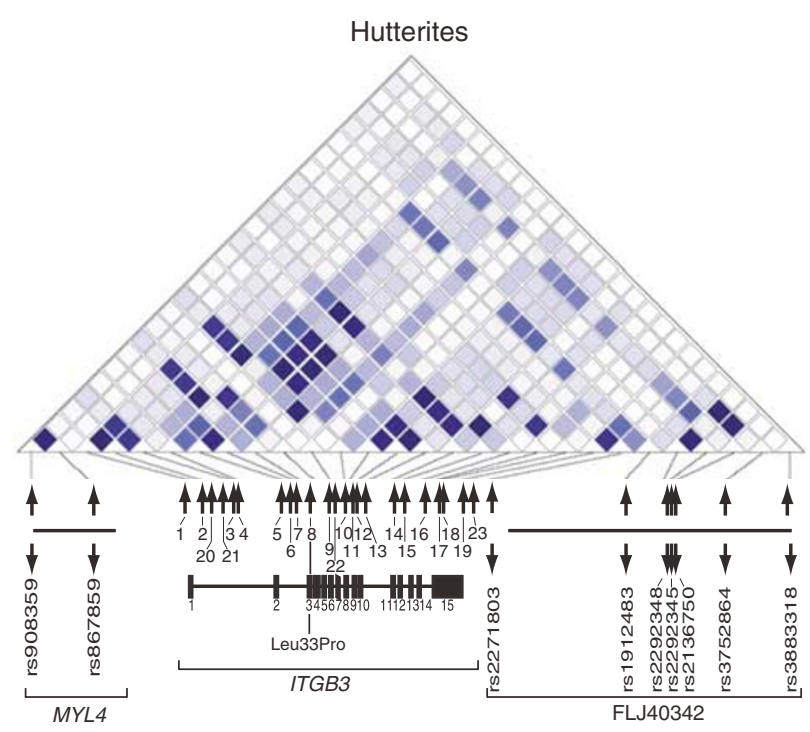

$r^{2} \quad 0.00 \quad 0.10 \quad 0.200 .30 \quad 0.40 \quad 0.50 \quad 0.60 \quad 0.700 .800 .90 \quad 1.00$

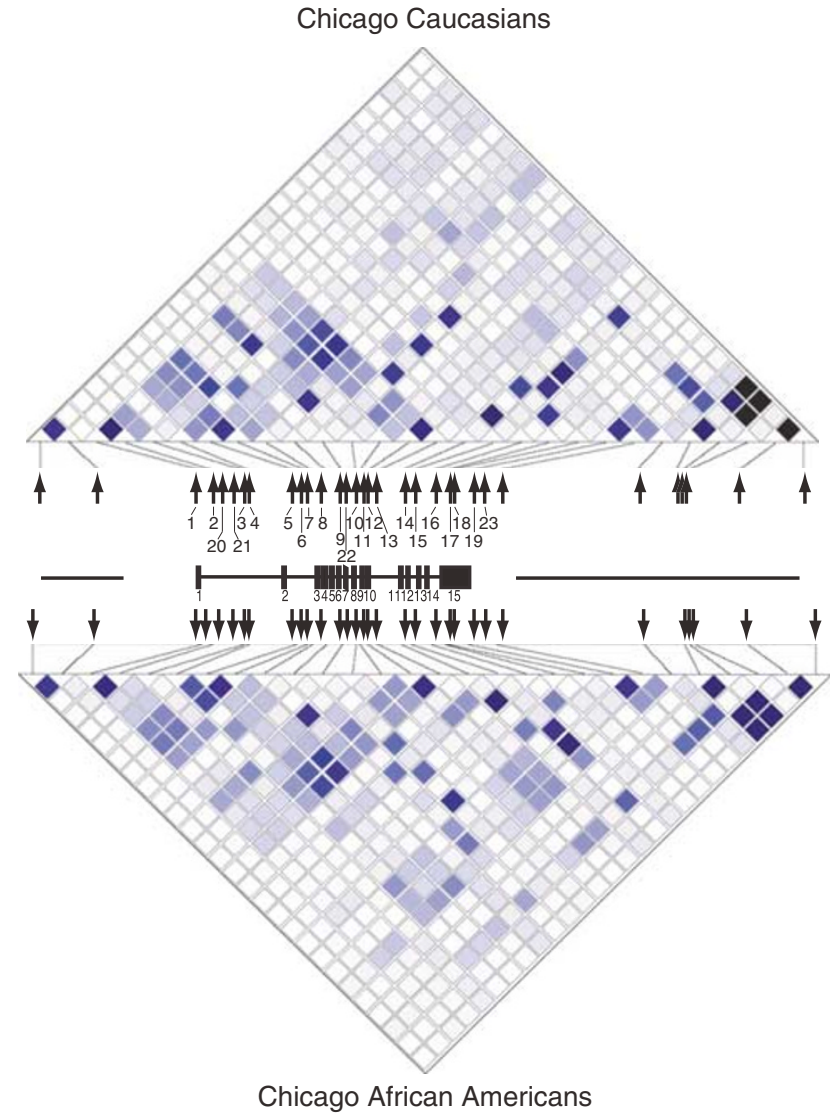

Figure 2 Intermarker LD $\left(r^{2}\right)$ plots for three populations. Each square represents pairwise LD measures $\left(r^{2}\right)$ between markers, with darker shading reflecting stronger correlations. ITGB3 SNP numbers are indicated with arrows below the upper LD graphics and shown relative to ITGB3 gene structure. The dbSNP identifiers for SNPs in flanking loci are indicated below the Hutterite plot, with a corresponding set of arrows, and presence within MYL4 or FLJ40432 gene loci. The position of Leu33Pro (SNP 8) is highlighted below the SNP numbers.

Although different polymorphisms were most associated in the three samples, estimates for the effect size of ITGB3 variants on whole-blood serotonin were remarkably similar across populations and SNPs. For each population, we estimated effect size using the most strongly associated SNP by single-point analysis: SNP 14 in the Hutterites (data not shown; method according to Abney et $a l^{34}$ ), SNP 1 in the Chicago sample, and SNP 11 in the autism sample. Although using the most significantly associated SNP may result in a slight upwards bias in effect size, the estimates still provide valuable information. In the male Hutterites, genotype at SNP 14 explained $6.8 \%$ of the variance in serotonin levels; heterozygotes at this SNP had a $12.7 \%$ increase in serotonin levels compared to homozygotes for the common allele. Effect size in homozygotes for the rare allele could not be accurately estimated, because there were too few individuals with this genotype $(N=4)$. Among outbred controls, genotype at SNP 1 explained $7.7 \%$ of the variance in serotonin levels; heterozygotes had a $17.4 \%$ increase in serotonin level in our additive model compared with homozygotes for the common allele. In the autism sample, genotype at SNP 11 explained $9.7 \%$ of the variance in serotonin levels; heterozygotes had a $17.0 \%$ increase in serotonin level compared with homozygotes for the common allele. These three SNPs are not in LD in the Hutterites or Chicago controls $\left(r^{2}<0.2\right)$.

\section{A coding SNP in ITGB3 is associated with autism, with} different effects in male and female subjects

The ultimate aim of these studies was to determine whether alleles at ITGB3 that were associated with serotonin levels were also associated with autism. We first performed a TDT to test for joint linkage and association in 87 Chicago autism trios, 50 of whom were included in the serotonin studies described above. The common allele (Leu) at ITGB3 SNP 8 (frequency 0.88) was modestly overtransmitted to autism probands at a 3:2 ratio, but was not statistically significant in this small sample $(\mathrm{T}=21$ vs $\mathrm{NT}=14 ; P=0.24$; Table 1 ). This was the highest transmission ratio among the five SNPs tested, including SNP $11(\mathrm{~T}=41$ vs NT=33; $P=0.35$; Table 1$)$, which was 
Table 1 Association results with autism: (a) results in the Chicago sample by the TDT and (b) results in the Vanderbilt sample by FBAT. ${ }^{36}$

\begin{tabular}{|c|c|c|c|c|c|c|}
\hline \multicolumn{7}{|c|}{ (a) Chicago autism sample } \\
\hline ITGB3 SNP & Allele & Transmitted & Nontransmitted & $\chi^{2}$ & \multicolumn{2}{|l|}{ P-value } \\
\hline $\begin{array}{l}\text { SNP } 1 \\
\text { SNP } 8^{a} \\
\text { SNP } 11 \\
\text { SNP } 14 \\
\text { SNP } 18\end{array}$ & $\begin{array}{c}\mathrm{G} \\
\mathrm{T} \text { (Leu) } \\
\mathrm{A} \\
\mathrm{T} \\
\mathrm{A}\end{array}$ & $\begin{array}{l}22 \\
21 \\
41 \\
13 \\
12\end{array}$ & $\begin{array}{r}22 \\
14 \\
33 \\
10 \\
9\end{array}$ & $\begin{array}{l}0 \\
1.4 \\
0.86 \\
0.39 \\
0.43\end{array}$ & $\begin{array}{l}1.0 \\
0.24 \\
0.35 \\
0.53 \\
0.51\end{array}$ & \\
\hline \multicolumn{3}{|c|}{ (b) Vanderbilt autism sample - FBAT - additive model } & $T_{O B S E R V E D}$ & $T_{\text {EXPECTED }}$ & & P-value \\
\hline $\begin{array}{ll}\text { SNP } & 1 \\
\text { SNP } & 20 \\
\text { SNP } & 21 \\
\text { SNP } & 8^{\mathrm{a}} \\
\text { SNP } & 22 \\
\text { SNP } & 11 \\
\text { SNP } & 16 \\
\text { SNP } & 23\end{array}$ & $\begin{array}{c}\text { T } \\
T \\
\text { G } \\
\text { T (Leu) } \\
\text { G } \\
\text { C } \\
\text { G } \\
\text { A }\end{array}$ & $\begin{array}{r}262 \\
385 \\
366 \\
205 \\
95 \\
211 \\
299 \\
410\end{array}$ & $\begin{array}{l}312 \\
561 \\
822 \\
554 \\
258 \\
356 \\
593 \\
622\end{array}$ & $\begin{array}{l}302 \\
554 \\
811 \\
523 \\
251 \\
355 \\
582 \\
621\end{array}$ & $\begin{array}{l}0.89 \\
0.50 \\
0.74 \\
3.1 \\
1.1 \\
0.067 \\
0.84 \\
0.054\end{array}$ & $\begin{array}{l}0.37 \\
0.62 \\
0.46 \\
0.0017 \\
0.29 \\
0.95 \\
0.40 \\
0.96\end{array}$ \\
\hline
\end{tabular}

FBAT, family-based association test; SNP, single-nucleotide polymorphism; TDT, transmission disequilibrium test.

${ }^{\mathrm{a}}$ In the combined sample, by FBAT, $P=0.00082$.

Table 2 FBAT analysis of Leu33Pro

\begin{tabular}{|c|c|c|c|c|c|c|c|c|c|c|c|c|c|c|}
\hline \multirow[b]{2}{*}{ Allele } & \multicolumn{5}{|c|}{ Overall } & \multicolumn{5}{|c|}{ Male susbjects } & \multicolumn{4}{|c|}{ Female subjects } \\
\hline & Fam \# & $T_{\text {OBSERVED }}$ & $T_{\text {EXPECTED }}$ & $Z$ & $P$ & Fam \# & $T_{\text {OBSERVED }}$ & $T_{\text {EXPECTED }}$ & $Z$ & $P$ & Fam \# & $T_{\text {OBSERVED }}$ & $T_{\text {EXPECTED }}$ & Z \\
\hline \multicolumn{15}{|c|}{ (a) Additive } \\
\hline Pro & 205 & 178 & 209.3 & -3.139 & 0.0017 & 186 & 156 & 169.3 & -1.502 & 0.133 & 77 & 31 & 49.0 & -3.6780 .000235 \\
\hline Leu & 205 & 554 & 522.7 & 3.139 & 0.0017 & 186 & 424 & 410.7 & 1.502 & 0.133 & 77 & 143 & 125.0 & 3.6780 .000235 \\
\hline \multicolumn{15}{|c|}{ Additive - considering linkage } \\
\hline Pro & 139 & 119 & 150.3 & -2.945 & 0.0032 & 129 & 107 & 120.3 & -1.465 & 0.143 & 69 & 24 & 42.0 & -3.3680 .00076 \\
\hline Leu & 139 & 469 & 437.7 & 2.945 & 0.0032 & 129 & 359 & 345.7 & 1.465 & 0.143 & 69 & 128 & 110.0 & 3.3680 .00076 \\
\hline \multicolumn{15}{|c|}{ (b) Dominant } \\
\hline Pro & 202 & 167 & 191.7 & -2.671 & 0.0076 & 182 & 144 & 150.7 & -0.822 & 0.411 & 76 & 27 & 45.0 & -3.9480 .000079 \\
\hline Leu & 27 & 40 & 33.4 & 2.243 & 0.0249 & 24 & 34 & 27.4 & 2.449 & 0.014 & 9 & $* \star \star \star * *$ & & \\
\hline \multicolumn{15}{|c|}{ Dominant - considering linkage } \\
\hline Pro & 145 & 123 & 147.7 & -2.512 & 0.0120 & 136 & 110 & 116.7 & -0.805 & 0.421 & 70 & 22 & 40.0 & -3.7120 .00021 \\
\hline Leu & 27 & 61 & 54.4 & 2.212 & 0.0270 & 24 & 43 & 36.4 & 2.464 & 0.014 & 9 & $\star \star \star \star \star *$ & & \\
\hline
\end{tabular}

FBAT, family-based association test; SNP, single-nucleotide polymorphism.

FBAT analysis of SNP 8 (Leu33Pro) was performed to test for differences in transmissions based on sex, and to test for potential differences based on the inheritance model. Analyses were performed with and without consideration of linkage at 17q21.3. Number of informative families (Fam \#), observed ( $\left.T_{\text {OBSERVED }}\right)$ and expected ( $\left.T_{\text {EXPECTED }}\right)$ transmissions, $Z$ scores, and $P$-values are indicated. Transmissions and statistics are shown separately for the overall combined sample, to male subjects, and to female subjects. The number of informative families was too small to allow a valid test of the Leu allele under a dominant model.

${ }_{* * * *}$ Indicates insufficient transmissions for a valid test.

associated with serotonin level in autism probands. A sexspecific analysis of this sample was not performed, as there were too few informative transmissions to female subjects (69 affected male and 18 affected female subjects).

We next genotyped a larger sample from Vanderbilt (643 largely multiplex families) for eight SNPs across ITGB3. In these families, the Leu allele at SNP 8 (frequency 0.87) was significantly overtransmitted to affected individuals (FBAT, $T_{\text {EXPECTED }}=523$ vs $T_{\text {OBSERVED }}=555 ; P=0.0017$; Table 1 ). When we combined the two autism samples, the associa- tion became more significant (FBAT, $P=0.00082$ ). These results remained very significant when the FBAT analysis considered the presence of linkage in the region $(P=0.00076$; Table 2).

Surprisingly, when we examined transmission to affected males $v$ females, the association between ITGB3 Leu33 and autism was stronger in female subjects ( $T_{\text {EXPECTED- }}$ MALE $=410$ vs $T_{\text {OBSERVED-MALE }}=424 ; P=0.13 ; T_{\text {EXPECTED- }}$ FEMALE $=125 v s T_{\text {OBSERVED-FEMALE }}=143 ; P=0.00024$ ), under an additive model (Table $2 \mathrm{a}$ ). Further characterization of 
Table 3 Gene-sex interaction analysis for ITGB3 and autism

\begin{tabular}{lccc}
\hline $\begin{array}{l}\text { Sample } \\
\text { (affecteds) }\end{array}$ & $R R$ (Leu/Pro) & $R R$ (Leu/Leu) & P-value \\
\hline Female (77) & 1.0 & 2.7 & $<0.0001$ \\
Male (267) & 4.0 & 4.0 & 0.0066 \\
& $\begin{array}{l}\text { Test of overall effect of } \\
\text { genotype }\end{array}$ & 0.0001 \\
& $\begin{array}{l}\text { Test of equal effects of } \\
\text { genotype across sexes }\end{array}$ & 0.0018 \\
\hline
\end{tabular}

The relative risk estimates in the best-fitting model are shown for the affected female and male samples relative to the Pro/Pro genotype. The test of overall effects using a general model (allowing for unequal genotype effects across samples) and a test of equal genotype effects across male and female samples are shown at the bottom. ${ }^{38}$

the association using FBAT to test transmissions under a dominant model suggests that this variant has reciprocal dominant effects in male and female subjects (Table 2b). Analysis by FBAT in male subjects shows a dominant risk effect of the Leu33 allele $\left(T_{\text {EXPECTED-MALE }}=27 v s T_{\text {OBSERVED- }}\right.$ MAle $=34 ; \quad P_{\text {MAle }}=0.014 ;$ Table $2 b$ ). In females, however, the Pro33 allele has a dominant protective effect $\left(T_{\text {EXPeCted-Female }}=45\right.$ vs $T_{\text {ObServed-Female }}=27 ; P_{\text {Female }}=$ $7.9 \times 10^{-5}$ ). To further evaluate these sex differences, we employed MODEL, which fits genetic models based on TDT information. ${ }^{38}$ This analysis indicates that both male and female subjects show a significant effect at this locus $\left(P_{\text {MAle }}=0.0066, P_{\text {Female }}<0.0001\right)$, but that the effects in these two samples are significantly different, confirming a gene-by-sex interaction $(P=0.0018)$. In agreement with FBAT analyses, MODEL shows that female data best fit a recessive risk effect, whereas male data best fit a dominant risk effect of the Leu33 allele (Table 3).

\section{Discussion}

ITGB3, a gene identified as a male QTL for whole-blood serotonin levels in the Hutterite founder population, is also a QTL for serotonin in outbred populations. In three samples, serotonin levels were associated with noncoding SNPs that account for $7-10 \%$ of the population variance in their respective samples. Extrapolating from studies of quantitative traits in model organisms in which most QTLs have effect sizes less than $5 \%,{ }^{39}$ we predict that the ITGB3 effect sizes observed in our study contribute substantively to interindividual variation in serotonin level in multiple populations.

Surprisingly, noncoding polymorphisms were more strongly associated with serotonin level than Leu33Pro in the Hutterites, Chicago controls, and the Chicago autism sample. A possible explanation is that one or more untyped variants are responsible for serotonin level and/or autism association, and the current association signals are the results of LD with markers we have not genotyped. However, we consider this unlikely because the SNPs associated in each sample were not in strong LD with each other. Alternatively, it is possible that more than one polymorphism at the ITGB3 locus has functional consequences. For example, noncoding variants could affect transcription or RNA processing, and this gene could show pleiotropy, such that effects on autism and serotonin levels are independent and influenced by different variation, perhaps intersecting in a common pathway. This would be consistent with our data, and with the various functional roles and locations of integrins, including effects on transcription and cell-cell signaling.

We were very surprised to find that the common Leu33 allele, which is associated with lower serotonin levels in the Hutterites, was associated with autism. Male-biased autism is known for its correlation to relatively higher serotonin levels in $\sim 30 \%$ of patients. How can we resolve these findings? First, the association between Leu33 and autism is not likely to explain the hyperserotonemia in autism. It is notable, however, that more patients are responsive to SSRI medications $(\sim 50 \%)$ than are hyperserotonemic. ${ }^{40}$ Therefore, we cannot exclude the possibility that even within the normal range of platelet serotonin levels, perturbations of the broader serotonin system (eg centrally) due to variation at ITGB3 might contribute to autism susceptibility. The functions of integrin $\beta 3$ have been most extensively studied in platelets; however, two previous findings should be noted. First, it has been hypothesized that high peripheral serotonin levels might influence central serotonin by feedback mechanisms before formation of the blood-brain barrier. ${ }^{41}$ Second, integrins, including $\beta 3$, have been shown to be critical to synapse maturation in the hippocampus, ${ }^{42}$ suggesting not only expression but also vital function in the developing brain.

Both male and female subjects show evidence for association of the Leu33 allele to autism; however, our data suggest that the allele has different effects on autism risk in male and female subjects, with the best-fitting models suggesting a dominant effect in male subjects and recessive effect in female subjects. We cannot exclude the possibility that the apparent difference in autism risk for male and female subjects is a chance finding. There could also be genetic modifiers or interactions that differ by sex, and failing to model for them could cause the main effects at ITGB3 to look different by sex. Many important questions remain, including the effect of Leu33Pro and other ITGB3 variation on serotonin levels by sex in large samples of control and autism subjects. In addition, the association of Leu33 with decreased serotonin level in the Hutterites (when hyperserotonemia is associated with autism) highlights the need for further investigation of our findings. 
An association with autism is particularly interesting, as chromosome 17q23 was not identified as a linkage region in autism in the 12 genome screens performed before its identification as a QTL for serotonin levels. ${ }^{35-45}$ However, two recent genome-wide studies report suggestive to significant evidence for linkage to autism in this region, ${ }^{43,44}$ and a meta-analysis indicates $17 \mathrm{q} 22-\mathrm{q} 23$ as significant at $P<0.05 .{ }^{45}$ Our findings support the idea that teasing apart a quantitative trait (whole-blood serotonin level), likely to be related to the etiology of a complex disease, can be useful in identifying candidate loci for subsequent analysis.

\section{Acknowledgements}

We acknowledge Dr Nancy Cox, Dr Shaun Purcell, and Dr Conrad Gilliam for helpful discussions and comments on this manuscript, and Kathy Hennessy, Natasha Phillips, Rebecca Anderson, Shuya Yan, and Zhi-Ying Yang for technical assistance. This research was supported by the University of Chicago Clinical Research Center (M01 RR00055), P5O HL56399, R01 HL66533, and R01 HL72414 to CO, U19 HD35482 to EC, MH061009 and NSO49261 to JS, and Vanderbilt Kennedy Center (P30 HD15052) Family Research and Hobbs/Marino Discovery grants to JS. LAW was supported by a National Science Foundation graduate research fellowship. We also acknowledge the invaluable assistance of the Vanderbilt University Center for Human Genetics Research DNA Resources and Computational Genomics Cores. Most importantly, we thank the families who have participated and contributed to these studies.

Biomaterials and phenotypic data provided through the NIMH Center for Collaborative Genetic Studies - Autism Genetics Initiative came from five projects: (1) Data and biomaterial collection was supported by NIH Grant MH55135 ('Collaborative Linkage Study of Autism') to Susan E Folstein, and her key Clinical and Phenotypic Coordinators were Brian Winklosky and Beth Rosen-Sheidley, MS, CGC. CoInvestigators included James S Sutcliffe and Jonathan L Haines. (2) Data and biomaterial collection was also supported by a supplement to NIH Grant MH61009 ('Molecular Genetics of 15q11-q13 Defects in Autism') and by Development Funds from the Vanderbilt Centers for Human Genetics Research and Kennedy Center for Research on Human Development to James S Sutcliffe. The Clinical and Phenotypic Coordinator for this project was Genea Crocket, MS. (3) Data and biomaterial collection from the Autism Genetic Resource Exchange (AGRE) collection was supported by NIH Grant MH64547 to Daniel H Geschwind, MD, PhD (UCLA) and the Cure Autism Now Foundation. Co-Principal Investigators include Stanley F Nelson, MD and Rita Cantor, PhD (UCLA), Christa Lese Martin, PhD (U Chicago), and T Conrad Gilliam, PhD (U Chicago). Co-investigators include Maricela Alarcon, PhD, Kenneth Lange, PhD, and Sarah J Spence, MD, PhD (UCLA), David H Ledbetter, PhD (Emory), and Hank Juo, MD, PhD (Columbia). (4) Data and biomaterial collection from Stanford was supported by NIMH Grant MH52708 to Neil Risch, PhD and Richard M Myers, PhD as Co-Principal Investigator. The Clinical Director in charge of recruitment and diagnostic data collection was Donna Spiker, PhD, in collaboration with Linda L Lotspeich, MD and Helena $C$ Kraemer, PhD. Earlier work at Stanford University on the genetics of autism was begun under the direction of Roland D Ciaranello, MD (deceased in December 1995). (5) Data and biomaterial collection from the University of Iowa was supported by NIMH Grant MH55284 to Joseph Piven, MD. Principal collaborators were Thomas Wassink, $M D$, Veronica Vieland, PhD, and Val Sheffield, MD, PhD.

\section{Electronic-database information}

dbSNP http://www.ncbi.nlm.nih.gov/SNP/

DNAprint http://www.dnaprint.com/

HGP http://www.genome.ucsc.edu

Innate immunity http://innateimmunity.net/IIPGA2/Bioinformatics/ MODEL http://pngu.mgh.harvard.edu/ purcell

VISTA http://pga.lbl.gov/

\section{References}

1 Kanner L: Autistic disturbance of affective contact. Nervous Child 1943; 2: 217-250.

2 Fombonne E: The prevalence of autism. JAMA 2003; 289: 87-89.

3 Chakrabarti S, Fombonne E: Pervasive developmental disorders in preschool children: confirmation of high prevalence. Am J Psychiatry 2005; 162: 1133-1141.

4 Wassink TH, Brzustowicz LM, Bartlett CW, Szatmari P: The search for autism disease genes. Ment Retard Dev Disabil Res Rev 2004; 10 : $272-283$.

5 Schain RJ, Freedman DX: Studies on 5-hydroxyindole metabolism in autistic and other mentally retarded children. J Pediatr 1961; 58: $315-320$.

6 Cook EH, Leventhal BL: The serotonin system in autism. Curr Opin Pediatr 1996; 8: 348-354.

7 Piven J, Tsai GC, Nehme E, Coyle JT, Chase GA, Folstein SE: Platelet serotonin, a possible marker for familial autism. J Autism Dev Disord 1991; 21: 51-59.

8 Cook Jr EH, Leventhal BL, Heller W, Metz J, Wainwright M, Freedman DX: Autistic children and their first-degree relatives: relationships between serotonin and norepinephrine levels and intelligence. J Neuropsychiatry Clin Neurosci 1990; 2: 268-274.

9 Leventhal BL, Cook Jr EH, Morford M, Ravitz A, Freedman DX: Relationships of whole blood serotonin and plasma norepinephrine within families. J Autism Dev Disord 1990; 20: 499-511.

10 Gordon CT, State RC, Nelson JE, Hamburger SD, Rapoport JL: A double-blind comparison of clomipramine, desipramine, and placebo in the treatment of autistic disorder. Arch Gen Psychiatry 1993; 50: 441-447.

11 Hollander E, Phillips A, Chaplin W et al: A placebo controlled crossover trial of liquid fluoxetine on repetitive behaviors in childhood and adolescent autism. Neuropsychopharmacology 2005; 30: $582-589$.

12 Sutcliffe JS, Delahanty RJ, Prasad HC et al: Allelic heterogeneity at the serotonin transporter locus (SLC6A4) confers susceptibility to autism and rigid-compulsive behaviors. Am J Hum Genet 2005; 77: $265-279$.

13 Devlin B, Cook EH, Coon $\mathrm{H}$ et al: Autism and the serotonin transporter: the long and short of it. Mol Psychiatry 2005; 10: $1110-1116$

14 Anderson GM, Gutknecht L, Cohen DJ et al: Serotonin transporter promoter variants in autism: functional effects and relationship to platelet hyperserotonemia. Mol Psychiatry 2002; 7: 831836.

15 Betancur C, Corbex M, Spielewoy C et al: Serotonin transporter gene polymorphisms and hyperserotonemia in autistic disorder. Mol Psychiatry 2002; 7: 67-71.

16 Persico AM, Pascucci T, Puglisi-Allegra $\mathrm{S}$ et al: Serotonin transporter gene promoter variants do not explain the hyperserotoninemia in autistic children. Mol Psychiatry 2002; 7: 795-800.

17 Coutinho AM, Oliveira G, Morgadinho T et al: Variants of the serotonin transporter gene (SLC6A4) significantly contribute to hyperserotonemia in autism. Mol Psychiatry 2004; 9: 264-271.

18 Weiss LA, Abney M, Cook Jr EH, Ober C: Sex-specific genetic architecture of whole blood serotonin levels. Am J Hum Genet 2005; 76: 33-41.

19 Weiss LA, Veenstra-Vanderweele J, Newman DL et al: Genomewide association study identifies ITGB3 as a QTL for whole blood serotonin. Eur J Hum Genet 2004; 12: 949-954. 
20 Weiss LA, Abney M, Parry R, Scanu AM, Cook Jr EH, Ober C: Variation in ITGB3 has sex-specific associations with plasma lipoprotein(a) and whole blood serotonin levels in a populationbased sample. Hum Genet 2005; 117: 81-87.

21 Weiss LA, Lester LA, Gern JE et al: Variation in ITGB3 is associated with asthma and sensitization to mold allergen in four populations. Am J Respir Crit Care Med 2005; 172: 67-73.

22 Hapke S, Gawaz M, Dehne K et al: beta(3)A-integrin downregulates the urokinase-type plasminogen activator receptor $(\mathrm{u}$ PAR) through a PEA3/ets transcriptional silencing element in the u-PAR promoter. Mol Cell Biol 2001; 21: 2118-2132.

23 Lindemann S, Tolley ND, Eyre JR, Kraiss LW, Mahoney TM, Weyrich AS: Integrins regulate the intracellular distribution of eukaryotic initiation factor $4 \mathrm{E}$ in platelets. A checkpoint for translational control. J Biol Chem 2001; 276: 33947-33951.

24 Riikonen T, Westermarck J, Koivisto L, Broberg A, Kahari VM, Heino J: Integrin alpha 2 beta 1 is a positive regulator of collagenase (MMP-1) and collagen alpha 1(I) gene expression. J Biol Chem 1995; 270: 13548-13552.

25 Goodall AH, Curzen N, Panesar M et al: Increased binding of fibrinogen to glycoprotein IIIa-proline33 (HPA-1b, PlA2, Zwb) positive platelets in patients with cardiovascular disease. Eur Heart I 1999; 20: 742-747.

26 Sajid M, Vijayan KV, Souza S, Bray PF: PlA polymorphism of integrin beta 3 differentially modulates cellular migration on extracellular matrix proteins. Arterioscler Thromb Vasc Biol 2002; 22: 1984-1989.

27 Vijayan KV, Goldschmidt-Clermont PJ, Roos C, Bray PF: The $\mathrm{Pl}(\mathrm{A} 2)$ polymorphism of integrin beta(3) enhances outside-in signaling and adhesive functions. J Clin Invest 2000; 105: 793-802.

28 Abney M, McPeek MS, Ober C: Estimation of variance components of quantitative traits in inbred populations. Am J Hum Genet 2000; 66: 629-650.

29 Ober C, Abney M, McPeek MS: The genetic dissection of complex traits in a founder population. Am J Hum Genet 2001; 69: 1068-1079.

30 Kim SJ, Cox N, Courchesne R et al: Transmission disequilibrium mapping at the serotonin transporter gene (SLC6A4) region in autistic disorder. Mol Psychiatry 2002; 7: 278-288.

31 Lord C, Rutter M, Le Couteur A: Autism Diagnostic InterviewRevised: a revised version of a diagnostic interview for caregivers of individuals with possible pervasive developmental disorders. J Autism Dev Disord 1994; 24: 659-685.

32 Lord C, Rutter M, Goode S et al: Autism diagnostic observation schedule: a standardized observation of communicative and social behavior. J Autism Dev Disord 1989; 19: 185-212.
33 Nickerson DA, Tobe VO, Taylor SL: PolyPhred: automating the detection and genotyping of single nucleotide substitutions using fluorescence-based resequencing. Nucleic Acids Res 1997; 25: $2745-2751$.

34 Abney M, Ober C, McPeek MS: Quantitative-trait homozygosity and association mapping and empirical genomewide significance in large, complex pedigrees: fasting seruminsulin level in the Hutterites. Am J Hum Genet 2002; 70 920-934.

35 Kruglyak L, Daly MJ, Reeve-Daly MP, Lander ES: Parametric and nonparametric linkage analysis: a unified multipoint approach Am J Hum Genet 1996; 58: 1347-1363.

36 Laird NM, Horvath S, Xu X: Implementing a unified approach to family-based tests of association. Genet Epidemiol 2000; 19 (Suppl 1): $\mathrm{S} 36-\mathrm{S} 42$

37 Lake SL, Blacker D, Laird NM: Family-based tests of association in the presence of linkage. Am J Hum Genet 2000; 67: $1515-1525$.

38 Purcell S, Cherny SS, Sham PC: Genetic Power Calculator: design of linkage and association genetic mapping studies of complex traits. Bioinformatics 2003; 19: 149-150.

39 Edwards MD, Stuber CW, Wendel JF: Molecular-marker-facilitated investigations of quantitative-trait loci in maize. I. Numbers, genomic distribution and types of gene action. Genetics 1987; 116: $113-125$.

40 McDougle CJ, Naylor ST, Cohen DJ, Volkmar FR, Heninger GR, Price LH: A double-blind, placebo-controlled study of fluvoxamine in adults with autistic disorder [see comments]. Arch Gen Psychiatry 1996; 53: 1001-1008.

41 Whitaker-Azmitia PM: Behavioral and cellular consequences of increasing serotonergic activity during brain development: a role in autism? Int J Dev Neurosci 2005; 23: $75-83$.

42 Chavis P, Westbrook G: Integrins mediate functional pre- and postsynaptic maturation at a hippocampal synapse. Nature 2001; 411: $317-321$.

43 Cantor RM, Kono N, Duvall JA et al: Replication of autism linkage: fine-mapping peak at 17q21. Am J Hum Genet 2005; 76: $1050-1056$

44 McCauley JL, Li C, Jiang L et al: Genome-wide and OrderedSubset linkage analyses provide support for autism loci on $17 \mathrm{q}$ and $19 \mathrm{p}$ with evidence of phenotypic and interlocus genetic correlates. BMC Med Genet 2005; 6: 1.

45 Trikalinos TA, Karvouni A, Zintzaras E et al.: A heterogeneitybased genome search meta-analysis for autism-spectrum disorders. Mol Psychiatry 2006; 11: 29-36.

Supplementary Information accompanies the paper on European Journal of Human Genetics website (http://www.nature.com/ejhg) 